\title{
Chemical stability and reversibility of PEDOT:PSS electrodes in view of low-cost biocompatible cellulose-assisted biosensors
}

\author{
H. Machrafi ${ }^{\mathrm{a}, \mathrm{b}}$, I. Bobinac ${ }^{\mathrm{a}}$, P. Dongo ${ }^{\mathrm{a}}$, V. Gallo ${ }^{\mathrm{a}}$, F. Iermano ${ }^{\mathrm{a}}$, C.S Iorio ${ }^{\mathrm{a}}$ \\ aPhysical Chemistry Group, Université libre de Bruxelles \\ ${ }^{\mathrm{b}}$ GIGA-In Silico Medicine, Université de Liège
}

\begin{abstract}
The increasing necessity for small electronics and wireless technologies in energetic devices, such as batteries or superconductors and in medical devices, such as sensors, drug delivery systems, calls for new materials, devices and preparation methods. In this work, the possibility of using a PEDOT:PSS hydrogel film as a cathode for a biocompatible cellulose-assisted biosensing device has been explored. The effect of film formulation and preparation method on the electrical conductivity is investigated, i.e. adding a cross-linker and different solvent additives on one side and adding an ionic liquid with and without a metal salt on the other side, for two film thicknesses. The chemical composition of the electrode is studied as a function of the used formulation as well as after being used as cathode in the biosensing device. It appeared that the electrode based on the cross-linker was mechanically robust, but suffered in electrical conductivity, despite the enhancement provided by the solvent additives. The electrode based on the ionic liquid showed a considerable increase in electrical performance, although the mechanical stability was not easy to maintain. The thin film showed a higher electrical conductivity. Although the metal salt enhanced somewhat further the electrical performance, the mechanical one suffered considerably. An application of the PEDOT:PSS electrode as cathode in a biosensing device showed promising results. Concentrations of the order of $\mu 1$ were measured without difficulty and the cathode seemed to be chemically stable and therefore reutilisable, opening future applications in reutilisable low-cost wearable biosensors and corresponding wearable batteries.
\end{abstract}

Keywords: PEDOT:PSS hydrogel, reversibility, biocompatible electrode, (bio)sensor cellulose

\section{Introduction}

The technological development of the last years has led to the birth of a new type of industry called "Industry 4.0". This term includes, amongst others, the Internet of Things (IoT), which is the extension of internet connectivity into physical devices and everyday objects. The Internet of Medical Things (IoMT) is an IoT application for medical and health-related purposes, data collection and analysis for research, and monitoring. Developments in plastic and electronics fabrication methods have enabled ultra-low-cost IoMT sensors [1]. The evolution of the IoMT devices aims towards the construction of micro devices. Implantable diagnosis/monitoring capsules, micro scale drug pumps, and implantable micro-sensing devices can be used as an example of miniaturized implanted medical devices (mIMDs) [2]. These tools usually require flexible, wearable, micro-sized, and even nontoxic, implantable power sources and sensors that can be easily integrated into the micro-devices, without inducing a significant size/weight increment. Such new type of smart and biocompatible devices could be used for wound healing biosensors and in general for bio-medical applications. An important element for such applications is the electrode and its biocompatibility. Besides biocompatibility, electric conductivity is an important feature for such electrodes. Traditional conductive materials used for these devices are metals such as $\mathrm{Au}, \mathrm{Cu}$, and steel, which have very high conductivity. Recently, conductive polymers (CPs) have attracted more and more attention owing to their excellent biocompatibility, chemical stability, low-temperature processing and especially flexibility. Conductive polymers are organic polymers that can be used as electrodes at the 
interface between the engineered device and the living organism. Their electrical properties can be fine-tuned using the methods of organic synthesis and by advanced dispersion techniques. The interest in CPs started with the discovery that polyacetylene (PA) can achieve high conductivity for an organic molecule [3]. Although this polymer was very well known by then, the research was not focused on the fact that in its regular state it expressed semiconducting behavior (conductivity $\sim 10^{-7} \mathrm{~S} \mathrm{~m}^{-1}$ compared to that of copper $\sim 10^{8} \mathrm{~S} \mathrm{~m}^{-1}$ ), just like most other conjugated polymers [4]. It was then found that PA can develop metal-like conductivity when exposed to iodine vapors $[5,6]$. This process of chemical oxidation by the halogen atom, also called doping, vapor was responsible for the increase in conductivity up to $10^{5} \mathrm{~S} \mathrm{~m}^{-1}$ [4]. This opened the path to the range of different conducting polymers of which polyaniline (PANI), polypyrrole (PPY) and poly-3,4-ethylene dioxythiophene (PEDOT) are attractive candidates in biomedical applications for their biocompatibility, ease of synthesis, low cost, and rich redox chemistry [4]. Among the various CPs, PEDOT is one of the most successful and mature materials under development [7-11]. PEDOT is a high conductivity polymer (of the order $O\left(10^{2}\right) \mathrm{S} / \mathrm{m}$ [12]), obtained by the chemical or electrochemical polymerization of EDOT monomers. For various applications, PEDOT needs to respond to aqueous media and show hydrogel properties. Therefore, it needs a counter-ion to be combined in polyelectrolyte complexes (PEC). The first compound to be used to form a PEC with PEDOT is polystyrene sulfonate (PSS), which is readily available on the market. As a dopant, the PSS is always used in excess in combination with the PEDOT. One of the ways to produce a fully stable PEDOT:PSS is by oxidative chemical polymerization of EDOT in the presence of PSS, using $\mathrm{Na}_{2} \mathrm{~S}_{2} \mathrm{O}_{8}$ as an oxidative agent $[13,14]$. This results into a PEDOT:PSS hydrogel that has the interesting property of swelling when in contact with water, electrolytes or biological fluids, allowing it to be applied as an electrode, membrane or sensor.

It is a well-known fact that the film morphology and chemical and physical structure strongly influence the electrical properties of PEDOT:PSS, which justifies quite some research in controlling and improving their properties [7-11]. Thus, a variety of post-treatments could be used to further improve the film performance. These methods are called secondary doping, given that these happen after the initial doping of the material. Using some of the treatments can bring PEDOT:PSS to perform on the level of indium tin oxide, the most widely used transparent conductive material [15]. Most of the secondary doping strategies could be divided into three categories [15]: thermal treatment, light treatment or chemical treatment. The latter is of particular interest in this paper and many different chemicals are used, such as organic solvents, ionic liquids, surfactants, salts, zwitterions, and acids. The shared characteristic is that most of these are done by simple solution mixing. However, the end-product may not always present mechanically stable features and result into brittle or uneven surfaces. This is important, since this work not only aims at assessing the electric properties and chemical stability, but it also focusses on the suitability of preparing an electrode and its application in biosensing devices. So, we explore here two approaches. The first is to start with a mechanically stable and robust PEDOT:PSS electrode by adding a cross-linker. Since it appears that this cross-linker reduces considerably the electric performances, a secondary chemical doping product is added in the form of an organic solvent during the preparation process, which will alter the polymers' structure and swelling, thereby enhancing their conductivity $[17,18]$. The second approach is to add a plasticizing, stabilizing and electrically enhancing secondary chemical doping agent to the PEDOT:PSS, i.e a low-temperature-melting salt, such as an ionic liquid (IL), which is an organic cation/inorganic anion salt with a primary defining characteristic of melting temperatures below $100{ }^{\circ} \mathrm{C}$. As a result of the charge screening effect of the IL, more crystalline and more interconnected PEDOT nanofibrillar structures are formed. This is the main reason behind the increase in conductivity induced by Ils, while an increase in stretchability is also observed [19]. These two approaches are not mixed in order to assess the outcomes of each 
approach. Finally, an application of the PEDOT:PSS electrode as a cathode in a biosensing device is demonstrated. Several characterizing methods and an overpotential model are used to investigate the electrical performance of the electrode as well its chemical stability and capability to serve in a biosensing device.

\section{Materials and Methods}

\subsection{Materials}

The main ingredient for the electrode preparation consists of an aqueous PEDOT:PSS dispersion CLEVIOS ${ }^{\mathrm{TM}}$ PH 1000, purchased from Heraeus, with a solid content between 1.0$1.3 \%$. Fig. 1 shows the chemical structure of the PEDOT and the PEDOT:PSS hydrogels. Two types of PEDOT:PSS electrodes are investigated. The first is based on the addition of an aqueous GOPS ((3-glycidyloxypropyl) trimethoxysilane) $50 \mu \mathrm{l} / \mathrm{ml}$ dispersion for stability enhancement as a cross-linker. As GOPS is reported [12] to significantly reduce the electric conductivity of PEDOT:PSS, electric enhancement additives are necessary. For this, the following organic solvents are used: Dimethylsulfoxide (DMSO), Polyethylene glycol diacrylate (PEG diacrylate) and ethanol. The second type of PEDOT:PSS electrode consists of using ionic salts as both stabilizers and electric enhancers. The ionic salt used for enhanced electric conductivity is 1-butyl-3-methylimidazolium octyl sulfate (ionic liquid, Sigma Aldrich, $\geq 96 \%$ HPLC). Additions of anhydrous copper(II) chloride $\left(\mathrm{CuCl}_{2}\right.$, Alfa Aesar, min $\left.98 \%\right)$ are reported to have little effect at low content, but increase electrical conductivity when added to PEDOT:PSS at high content [20]. However, we already chose to use an ionic salt because it not only increases the electrical conductivity, but serves in addition for stability enhancement. Therefore, we don't investigate the addition of $\mathrm{CuCl}_{2}$ to PEDOT:PSS, but instead are interested in the effect $\mathrm{CuCl}_{2}$ could have on ionic-liquid-doped PEDOT:PSS. The electrolyte used for the cyclic voltammetry test and for the sensor device is a filtered phosphate buffer solution (PBS), with $\mathrm{pH}$ 7.4, purchased from Sigma-Aldrich. The separator for the sensing device is a Whatman $^{\circledR}$ cellulose chromatography $1 \mathrm{Chr}$ paper, purchased from Sigma-Aldrich. The graphene dispersion is bought from Cambridge University.

(a)

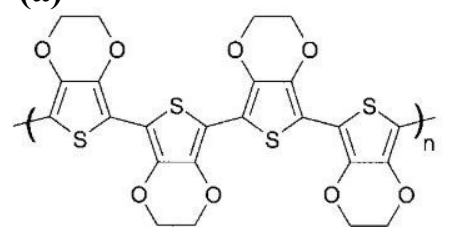

(b)

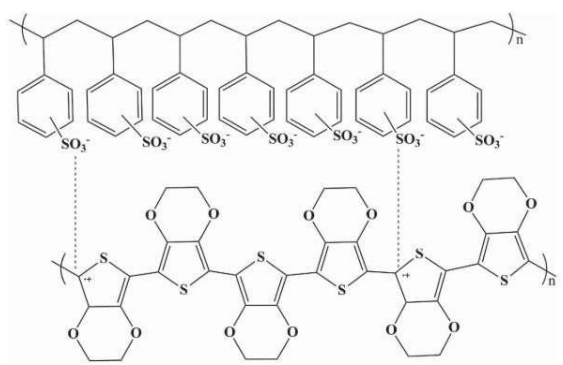

Figure 1. Chemical structure of (a) PEDOT [13] and (b) PEDOT:PSS [15]

\subsection{GOPS-based PEDOT:PSS electrode preparation}

It can be observed in Fig. 2 that without the addition of a cross-linker the dried PEDOT:PSS layer loses its mechanical stability (Fig. 2(a)), especially in an aqueous medium where it even disintegrates (Fig. 2(b)). Since for many applications, such as batteries or sensors, an aqueous medium is often used, mechanical stability is necessary. To enhance the stability in aqueous environments a polymer cross-linking agent, a $50 \mu \mathrm{l} / \mathrm{ml}$ GOPS ((3-glycidyloxypropyl) trimethoxysilane) solution was added. 
(a)

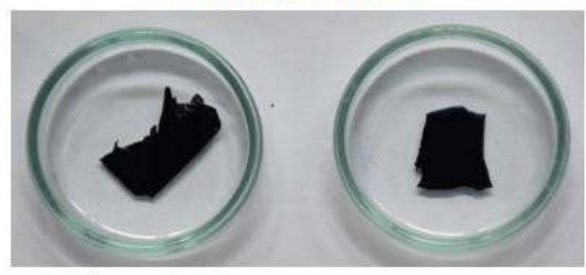

Without GOPS

With GOPS (b) In water

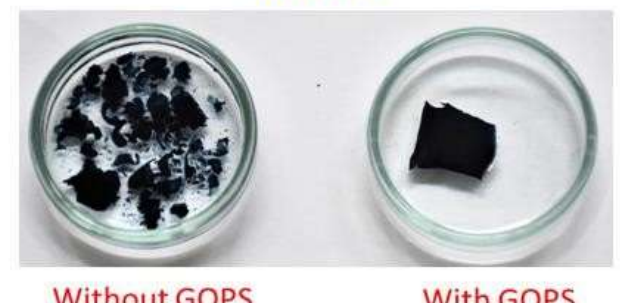

Figure 2. Difference between the layers of PEDOT:PSS with and without the addition of GOPS

The following procedure has been carried out to produce the electrode. First, $3 \mathrm{ml}$ of a previously sonicated aqueous PEDOT:PSS dispersion were introduced into a beaker with a pipette, adding to it $150 \mu \mathrm{l}$ of GOPS and the electric conductivity enhancer at the end. To enhance the electrical properties different additives have been considered. There, $300 \mu 1$ of the following electrical conductivity enhancers were added to the dispersion: dimethylsulfoxide (DMSO), polyethylene glycol diacrylate (PEG diacrylate) and ethanol. The dispersion was sonicated for 7 minutes to ensure adequate mixing of the additives, deposited with a pipette in a $1 \mathrm{~cm}^{2}$ square shape and left under the hood for 14 hours to evaporate the water.

\subsection{Ionic-liquid-based PEDOT:PSS electrode preparation}

A sonicated aqueous PEDOT:PSS dispersion is mixed with the ionic liquid 1-butyl-3methylimidazolium octyl sulfate. After mixing, $5 \mathrm{~g}$ of the mixture was poured into a $25 \mathrm{~cm}^{2}$ square and $5 \mathrm{~mm}$ deep Teflon mold. A mold is used in order to maintain a regular thickness. Drying lasted for 18 hours at room conditions. Annealing was done in a preheated oven at 130 ${ }^{\circ} \mathrm{C}$ for 25 minutes inspired from the procedure described in [19]. The ionic liquid content has been varied from 0 to $60 \mathrm{wt} \%$ of the final product. For the assessment of the effect of $\mathrm{CuCl}_{2}$ on an ionic-liquid-based PEDOT:PSS electrode, the ionic liquid content was fixed at $45 \mathrm{wt} \%$ and adding to it $\mathrm{CuCl}_{2}$ content up to $5 \mathrm{~mol} \%$. The obtained samples are assigned as "thick".

Since there is great interest in thin films, a thinner film is prepared by mixing the same components as mentioned above and pouring $1 \mathrm{~g}$ of the mixture on a PDMS substrate of $25 \mathrm{~cm}^{2}$ surface. A mold was not necessary here due to the much thinner film thickness. The deposited film is then left to dry for 4 hours at room conditions. Annealing was done in the oven at 130 ${ }^{\circ} \mathrm{C}$ for 20 minutes, with gradual increase of temperature from $20{ }^{\circ} \mathrm{C}$ to $130{ }^{\circ} \mathrm{C}$. These samples are assigned as "thin".

Both the thick and thin samples are, respectively, took out from their molds and delicately scraped off the substrate, before they are used as an electrode for further analysis or as a cathode for a sensing device.

\subsection{Sensor assembly}

Figs. 3(a) and (b) present a scheme for the used sensor assembly. A current is measured, induced by a deposited electrolytic liquid, during discharge, gouverned by an electrochemical metal-air reaction catalysed by the PEDOT:PSS hydrogel cathode. The level of the measured current should indicate the concentration of the electrolyte in the liquid. Although the PEDOT:PSS electrode is bendable in itself, for the moment, this aspect is not under investigation and the sensor is held between polylactic acid (PLA) support sheets. A particular geometry (Fig. 3(a)) with a groove for the sensor elements and holes to allow assembly was designed with the SolidWorks 2017 software and produced with the Ultimaker 33D printer. The grooves designed have a thickness of $0.4 \mathrm{~mm}$, which ensure good adhesion of the various components once assembled, allow the necessary passage of air and provides for terminals that can be connected to the measuring instruments. The doped PEDOT:PSS electrode serves as the cathode, while a 
$0.25 \mathrm{~mm}$ thick zinc ( $\mathrm{Zn}$ ) foil (Sigma-Aldrich) serves as an anode. A dry cellulose filter paper, with pore sizes from 20 to $25 \mu \mathrm{m}$, separates the electrodes. As graphene has a high conductivity, its addition to the separator appeared to be beneficial and improve ionic charge transfer. To prepare the graphene-coated separator, the chromatographic paper was immersed in a dispersion of $2 \mathrm{mg} / \mathrm{mL}$ graphene, purchased from Cambridge University, and left to dry out for 6 hours. To ensure a good connection, an $80 \mu \mathrm{m}$ thick gold foil was glued directly to the PLA supports and pressed, respectively, on both sides of the electrodes. A square hole was cut in the PEDOT:PSS electrode and in the PLA support at it's side, leaving the filter paper exposed. Finally, all these components were stacked together and then everything was held with Teflon screws. After the assembly of the sensor, it was connected to a Keithley 2400 SourceMeter SMU measuring instrument, a certain known amount of PBS is added on the exposed filter paper, triggering a current response and the discharge test was started and the current was measured.

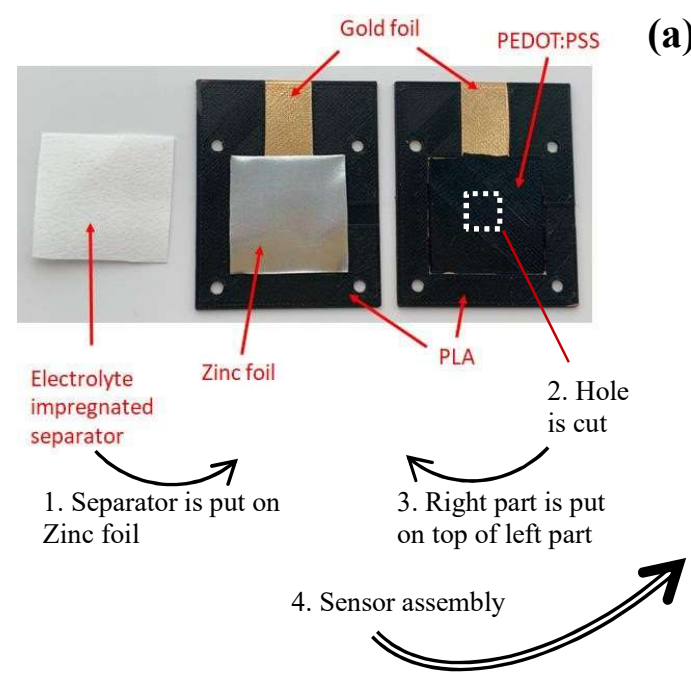

(a)

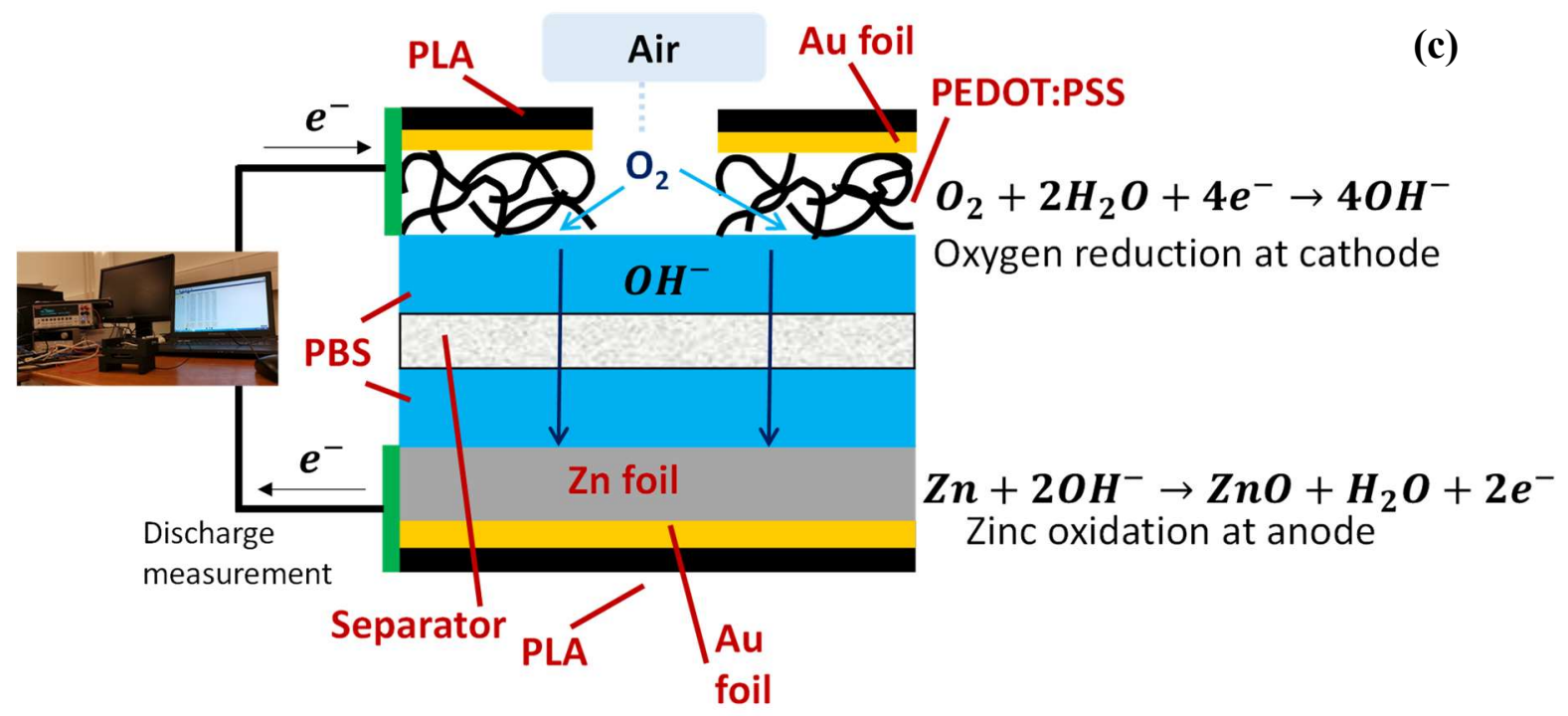

Figure 3. (a) Sensor assembly, (b) assembled biosensor, (c) schematic representation of the chemical reactions during electrolyte measurement by the biosensor

Fig. 3(c) shows the chemical process taking place during the sensing. When the separator is dry, the electric circuit is not closed, and nothing happens. As soon as the PBS is added on the separator it wets the space between the respective electrodes and the separator. This will make 
the PEDOT:PSS hydrogel swell, creating micro/nanopores for oxygen to diffuse through and enabling the oxygen reduction reaction. As such, the electric circuit is now closed, and a redox reaction starts to occur. The oxygen from the air is reduced at the PEDOT:PSS cathode, releasing hydroxyl ions that diffuse through the electrode towards the zinc anode where it oxydizes zinc to zinc oxide [21]. The reaction occurring at the PEDOT:PSS cathode is represented by

$\mathrm{O}_{2}+2 \mathrm{H}_{2} \mathrm{O}+4 e^{-} \rightarrow 4 \mathrm{OH}^{-}$,

with a standard cathode electrode potential at $25^{\circ} \mathrm{C}$ of $E_{c}^{0}=0.4 \mathrm{~V}$, whereas at the $\mathrm{Zn}$ anode the following reactions occur

$\mathrm{Zn}+4 \mathrm{OH}^{-} \rightarrow \mathrm{Zn}(\mathrm{OH})_{4}^{2-}+2 e^{-}$

$\underline{\mathrm{Zn}}(\mathrm{OH})_{4}^{2-} \rightarrow \mathrm{ZnO}+\mathrm{H}_{2} \mathrm{O}+2 \mathrm{OH}^{-}$

$\mathrm{Zn}+2 \mathrm{OH}^{-} \rightarrow \mathrm{ZnO}+\mathrm{H}_{2} \mathrm{O}+2 e^{-}$

with a standard anode electrode potential at $25^{\circ} \mathrm{C}$ of $E_{a}^{0}=-1.25 \mathrm{~V}$. The equilibrium standard potential is given by $E_{e q}=E_{c}^{0}-E_{a}^{0}=1.65 \mathrm{~V}$. It is easy to see that the total reaction is $2 Z n+$ $\mathrm{O}_{2} \rightarrow 2 \mathrm{ZnO}$. As $E_{e q}>0$, it is a spontaneous reaction, delivering a current. The measured current's value will change as the concentration of electrolytes in the PBS changes as this would increase charge transfer. As a side note, the working voltage on discharge will be lower than the theoretical $1.65 \mathrm{~V}$, due to the internal loss of the cell, ohmic and concentration losses (overpotential), being typically less than $1.2 \mathrm{~V}[21,22]$.

\section{Characterization techniques}

Four-probe resistance measurements, Cyclic Voltammetry, FTIR, SEM/EDS analyses, Profilometry, Digital Microscopy and overpotential calculation have been used to characterize the PEDOT:PSS.

\subsection{Four Probe resistivity measurement}

The primary technique for measuring sheet resistance is the four-probe method (also known as the Kelvin technique). The electrodes must transport electrical charge laterally and need low sheet resistances to reduce losses during this process. Furthermore, the resistivity and conductivity can be calculated if the sheet resistance and material thickness are known. This allows for the materials to be electrically characterized, purely by measuring their surface resistivity. A four-probe system consists of four electrical probes in a line, with equal spacing between each of the probes as shown in Fig. 4(a).

(a)

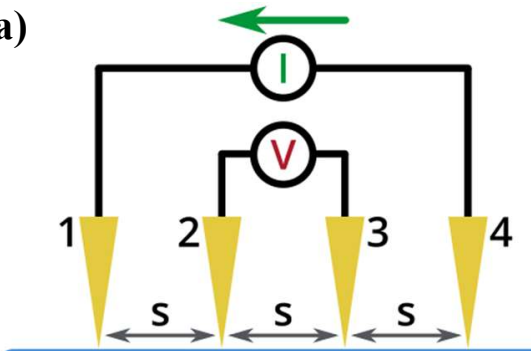

(b)

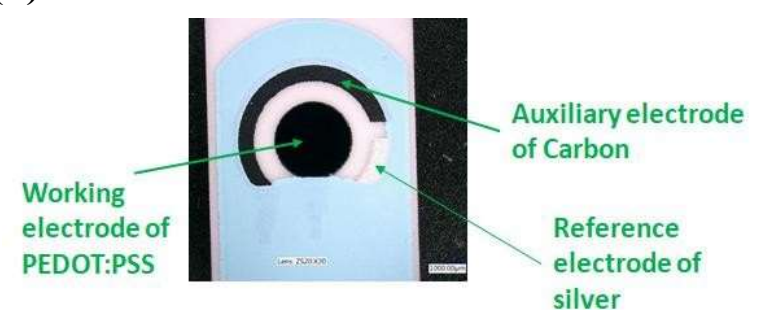

Figure 4. (a) Scheme of four-probe system for the measurement of the sheet resistance, (b) preprinted mini three-electrode system for the cyclic voltammetry tests 
The measurement of surface resistance with this four-probe system operates by applying a current $I$ on the outer two probes and measuring the resultant voltage drop $V$ between the inner two probes. For a uniform spacing $S=7 \mathrm{~mm}$ between the probes (with contact diameter of 0.4 $\mathrm{mm}$ ), and a sheet thickness much smaller than the probe spacing, the sheet resistance is given by $R_{S}=C \frac{\pi}{\ln 2} \frac{V}{I}$ [23], where we introduced a geometrical correction factor $C$. The geometrical correction factor equals unity when the sample dimensions (length $L$ and width $W$ ) are significantly larger than the probe spacing, i.e. $L \gg S$ and $W \gg S$ so that the sheet resistace is $R_{S} \equiv R_{S}^{\infty}$. By varying the length and width of the samples, measuring the sheet resistance and comparing it to $R_{S}^{\infty}$, a calibration test is performed. Knowing the electrode sample width and length, it can be interpolated that $C \approx 0.43$. As the sheet resistance is not a material property, it is more appropriate to speak in terms of the sheet resistivity $\varrho$ given by $\varrho=R_{S} \delta$, where $\delta$ is the sample thickness. The sample thickness is obtained by means of profilometry and digital microscopy. The surface electric conductivity is simply given by $\sigma=\varrho^{-1}$.

\subsection{Profilometry and Digital Microscopy}

A Veeco Dektak 150 Surface Profiler and a Keyence digital microscope VHX 6000 have been used to measure the sample thicknesses. The Veeco Dektak ${ }^{\circledR} 150$ Surface Profiler measures the surface topography by converting the vertical movement of a stylus in contact with the sample surface into an electrical signal [24]. The Keyence digital microscope vhx 6000 measures optically with high resolution the thickness of the sample layer that is pressed between two polycarbonate blocks.

\subsection{Cyclic Voltammetry}

The cyclic voltammetry (CV) experiments have been carried out using a pre-printed mini threeelectrode system on which the PEDOT:PSS electrode was printed with a Dimatix Materials Printer $D M P-2850$ to act as a working electrode, as shown in Fig. 4(b). A carbon counter electrode, a silver reference electrode and a phosphate-buffered saline (PBS) solution as the electrolyte have been used. This pre-printed cell was connected to the " $\mu$ Stat 400 bipotentiostat/galvanostat" measuring instrument and immersed in a beaker containing the electrolytic solution. The working electrode potential is ramped linearly versus time. After the set potential is reached in a CV experiment, the working electrode's potential is ramped in the opposite direction to return to the initial potential. The current at the working electrode is plotted versus the applied voltage (that is, the working electrode's potential) to give the cyclic voltammogram trace.

\subsection{FTIR Analyses}

FTIR analyzes have been performed on the PEDOT:PSS electrodes to detect the chemical bonding and changes at the surface of the structures. The samples were analyzed with a Jasco FT/IR-6600 FT-IR Spectrometer.

\subsection{SEM and EDS}

In this work, Hitachi $S U-70$ scanning electron microscope was used with accelerating voltage of $20 \mathrm{kV}$. Used magnifications were between 30 and 15000 times. Non-conductive samples were treated with gold using a low-vacuum sputter deposition. After interaction with primary electrons X-ray photons are generated. These are formed as a side effect of electron transition from a higher excited state to a lower one $[25,26]$. Signals created in this way could be analyzed in order to determine the chemical composition of materials, since every element will create its own distinct X-ray spectra. For this analysis, Energy Dispersive X-ray spectroscopy (EDS) [25] is performed using the Hitachi SU-70 machine. 


\subsection{Overpotential calculation for a nanoporous hydrogel cathode}

The overpotential due to concentration polarization at the cathode is given by [27]

$\eta_{c}=-\frac{R_{g} T}{4 F} \ln \left(1-\frac{i}{i_{c s}}\right)$

where $R_{g}, T, F, i$ and $i_{c s}$ is the universal gas constant, temperature, Faraday's constant, the actual current density and the limiting current density, respectively. The latter is given by [27]

$i_{c s}=\frac{4 F}{R_{g} T} \frac{D_{e}}{\delta} p_{0}\left(\frac{p}{p-p_{0}}\right)$

where $\delta, p_{0}$ and $p$ are the cathode thickness, oxygen partial pressure and the atmospheric pressure, respectively. Since the pores are of the order of magnitude of the mean free path of oxygen (which can be given by $\ell=\frac{k_{B} T}{4 \pi r_{O_{2}}^{2} p}$ with $k_{B}$ Boltzmann's constant and $r_{O_{2}}$ the radius of oxygen), the diffusion coefficient shows non-local effects, described in [28,29], so that it concerns an effective diffusion coefficient $D_{e}$. In [29], it has been established that non-local, non-Fickian, diffusion through nano/micro-pores can be described by the following constitutional equation:

$\boldsymbol{J}=-D \nabla c+\ell^{2} \nabla^{2} \boldsymbol{J}$

where $\boldsymbol{J}, D$ and $c$ are the diffusion flux, oxygen diffusion coefficient and oxygen concentration, respectively. In case of oxygen diffusion in the cathode, we can in a cylindrical pore configuration make the approximation $\nabla^{2} \boldsymbol{J} \approx-\frac{8}{r_{p}^{2}} \boldsymbol{J}$, where non-local Darcy flow is compared to an analogous Poiseuille flow with the corresponding effective property, as is shown in [30]. In this case, this effective property is the effective diffusion coefficient and it is of the form

$D_{e}=\frac{1}{1+8 K n_{p}^{2}} D$

with a Knudsen number $K n_{p}=\frac{\ell}{r_{p}}$. The pore size has not been determined in the literature and is not so straightforward to obtain. From SEM images and [31], we can estimate the pore size by $1 \mu \mathrm{m}$. The oxygen size being around $350 \mathrm{pm}$, the mean free path can be calculated to be around $100 \mathrm{~nm}$ at ambient temperature and pressure. This gives $K n_{p}=0.2$ and with $D=2 *$ $10^{-9} \mathrm{~m}^{2} / \mathrm{s}$, Eq. (4) gives $D_{e} \approx 1.5 * 10^{-9} \mathrm{~m}^{2} / \mathrm{s}$. With an electrode thickness of $370 \mu \mathrm{m}$, an oxygen partial pressure of $0.21 \mathrm{~atm}$, an electrode surface of $10^{-4} \mathrm{~m}^{2}$, (and with $F=$ $96485 \mathrm{~s} \frac{\mathrm{A}}{\mathrm{mol}}, R_{g}=8.3145 \frac{\mathrm{J}}{\mathrm{mol} \mathrm{K}}$ ), the limiting current density at ambient temperature is calculated to be $i_{c S} \approx 16.6 \mathrm{~A} / \mathrm{m}^{2}$. With this, Eq. (1) gives the overpotential as a function of the operating/measured current.

\section{Results and Discussion}

\subsection{GOPS-based PEDOT:PSS electrode}

\subsubsection{Electrode performance}

Fig. 5 shows an image of a GOPS-based PEDOT:PSS electrode, obtaining a smooth and stable sheet. 


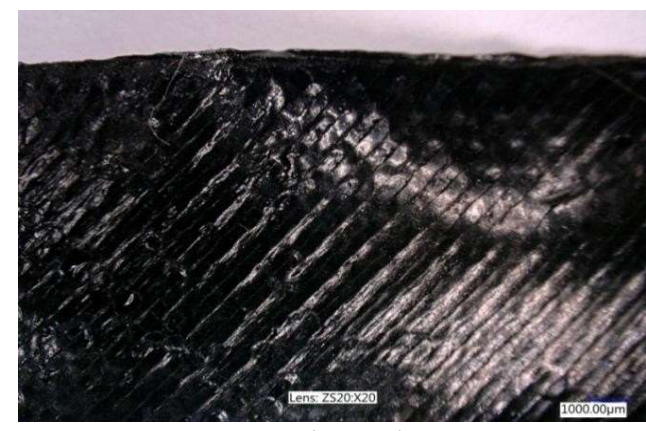

Figure 5. GOPS-based PEDOT:PSS electrode

The electrodes must transport electrical charge laterally and need low sheet resistance to reduce losses during this process. The resistivity and profilometry measurements are given in Table 1 for the GOPS-based PEDOT:PSS electrode with the three organic additives. The electric conductivities of pure PEDOT:PSS without and with GOPS (5 vol\%) are 322 and $0.004 \mathrm{~S} / \mathrm{m}$, respectively, corresponding approximately to reported values in [12]. While GOPS aids at producing robust and stable PEDOT:PSS electrodes allowing easy use in biosensing devices, their conductivity is quite low. The addition of organic solvent additives during the film formation process is necessary for its use as an electrode.

Table 1. Electric performance of the GOPS-based PEDOT:PSS electrodes for three different additives

\begin{tabular}{lllll}
\hline Additive & $R_{S}[k \Omega]$ & $\delta[\mu m]$ & $\varrho[\Omega m]$ & $\sigma[\mathrm{S} / \mathrm{m}]$ \\
\hline \hline DMSO & 20.2 & 370 & 7.5 & $0.13 \pm 0.01$ \\
PEG diacrylate & 22.3 & 450 & 10 & $0.10 \pm 0.01$ \\
Ethanol & 227 & 270 & 61 & $0.016 \pm 0.002$ \\
\hline
\end{tabular}

It can be seen from Table 1 that the additives increase the electric conductivity of the GOPSPEDOT:PSS electrode considerably, with DMSO even by two orders of magnitude. From the results obtained, the DMSO turns out to be the additive that gives the least resistivity to the PEDOT:PSS layer and therefore the most suitable for the production of a GOPS-based electrode. Although the electrical conductivity is on the low side, it will be shown later that it is sufficient for biosensing. For long-term, low current density applications, such an electrode can be quite apt. In applications, e.g. sensors or batteries, the polymer electrode (often used as the cathode) may undergo changes during the discharge process (see Appendix A) [32,33]. Cyclic voltammetry tests were performed to analyze the reversibility of the PEDOT:PSS electrode. This test was performed on the two most promising layers: with the addition of DMSO and PEG diacrylate. From these analyses, the CV curves were obtained in Fig. 6.

(a)

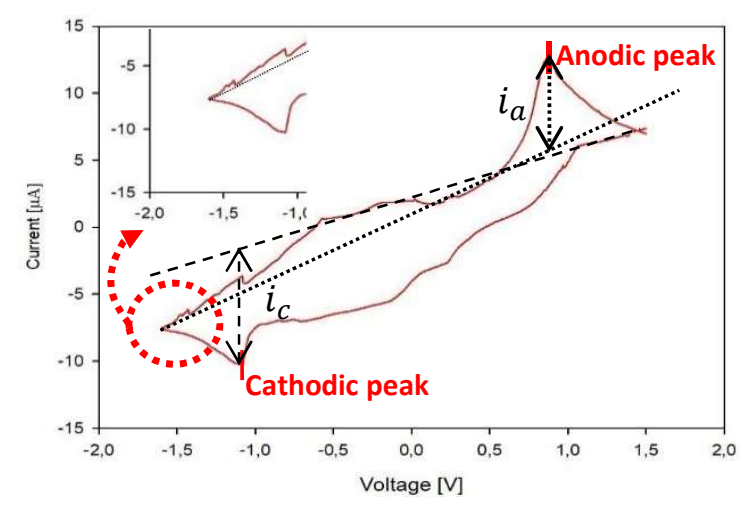

(b)

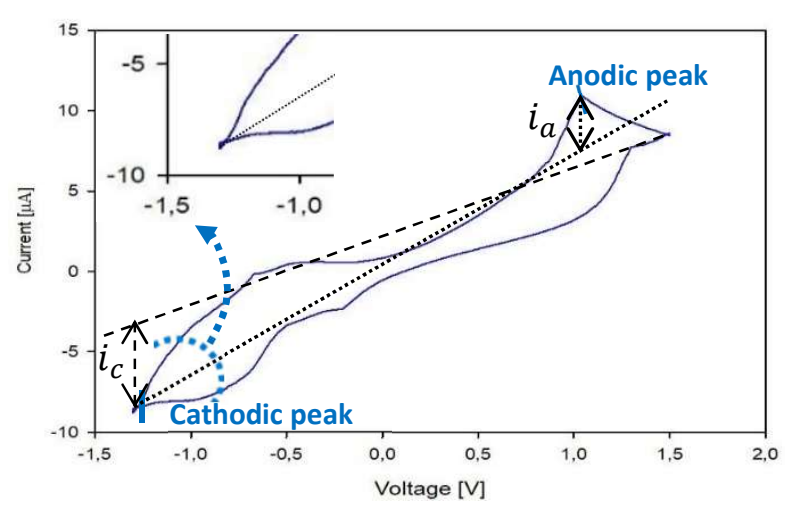


Figure 6. Cyclic voltammetry curves for the PEDOT:PSS hydrogel electrodes with the addition of (a) DMSO and (b) PEG diacrylate

The reversibility of the PEDOT:PSS electrodes can be deduced from the cyclic voltammograms in Fig. 6. For this, we need to estimate the cathodic and anodic peak currents, i.e. $i_{c}$ and $i_{a}$, respectively. The anodic peak currents can be determined by extrapolating, during the oxidative scan (increasing the voltage from negative to positive values), the relatively flat curve before its slope changes (defined as the onset potential for oxidation). The difference between the current at the anodic peak and that at the extrapolated curve (at the anodic peak potential) is defined as the anodic peak current. The same procedure is done in reverse, during the reductive scan (decreasing the voltage from positive to negative values), which gives the cathodic peak current. This procedure to determine the cathodic and anodic peak currents are illustrated in Fig. 6, where the definitions of cathode and anode correspond to a discharge case. The electrode is reversible when $\left|i_{c}\right| \equiv\left|i_{a}\right|$. As illustration, an example of an irreversible electrode, a graphite electrode, is presented in Appendix B. For the DMSO-doped electrode, we find that $\frac{\left|i_{a}\right|}{\left|i_{c}\right|} \approx 0.9$, while for the PEG-diacrylate-doped electrode, we find $\frac{\left|i_{a}\right|}{\left|i_{c}\right|} \approx 0.6$. This shows that the PEGdiacrylate-doped electrode is only partially reversible, while the DMSO-doped electrode can be considered reversible and motivates its use due to an expected reusability. Indeed, DMSO is also often the preferred reference additive for its wide and successfull applicability [34]. The question raised, though, is whether it does not interfere with the PEDOT:PSS's biocompatibility.

\subsubsection{Biocompatibility analysis of the layer with DMSO}

DMSO is considered a particular solvent as it is easily absorbed by the skin and can drag dangerous substances inside. However, it is often used in biocompatible layer preparations and it is reported to be able to induce orientation of the polymer chains of the PEDOT:PSS, allowing better conduction between the chains themselves [35,36]. The danger associated with the use of DMSO is in fact eliminated when its benefit is established but the substance itself is no longer active, either due to it having undergone a chemical reaction or having finally evaporated from the end product. This brings to attention whether in our electrode, DMSO is still present or not. To understand how DMSO can act on the layer and whether there is the presence of this substance even on the dry layer, FTIR analyses were conducted on layers with and without the addition of DMSO during the preparation of the electrode. Fig. 7(a) shows FTIR spectra of DMSO and of a DMSO-assisted GOPS-based PEDOT:PSS electrode. 

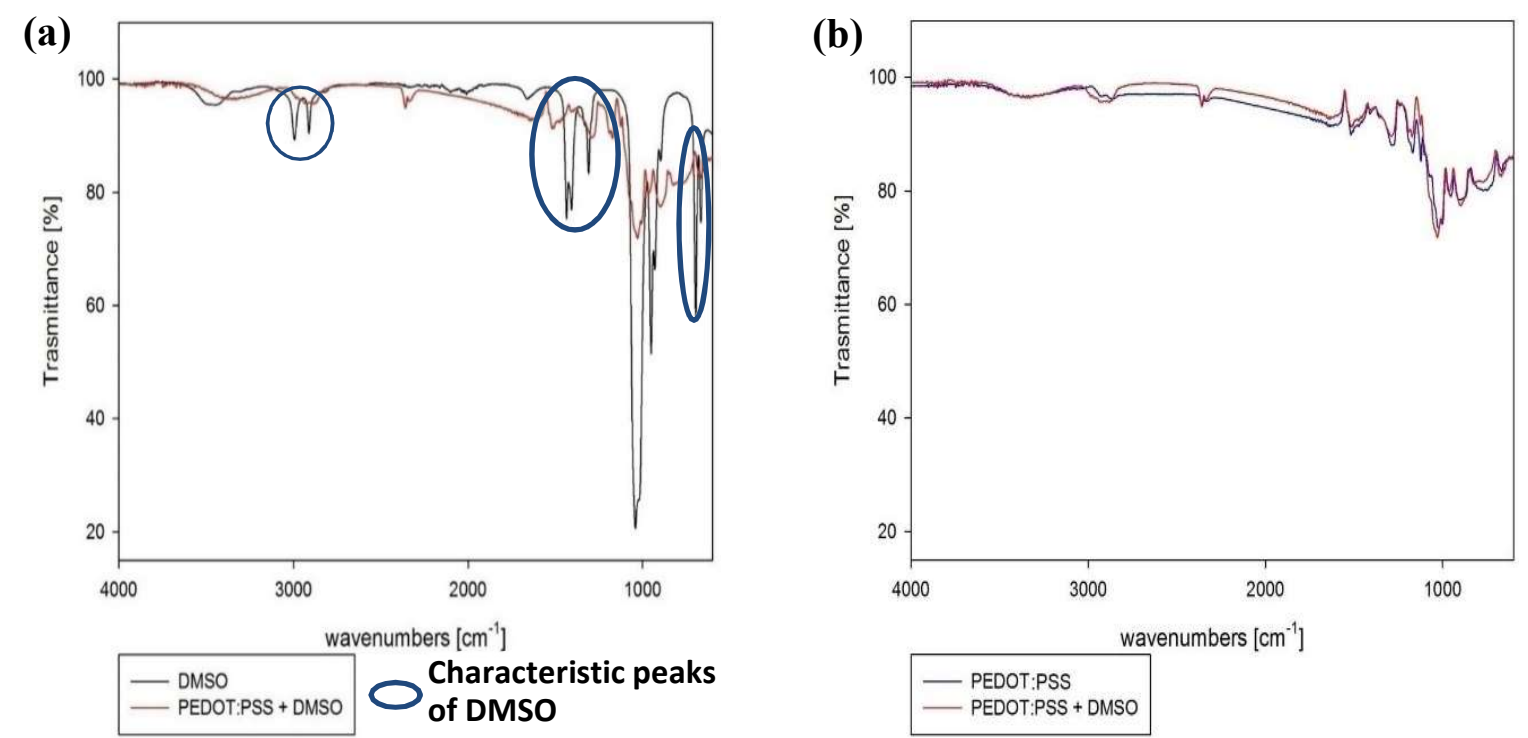

Figure 7. (a) Comparison between the spectrum of the dried layer with the addition of DMSO and that of pure DMSO, (b) comparison of the spectra of the dried layers with and without the addition of DMSO.

By comparing the spectrum of the dried layer with the addition of DMSO and the spectrum of the pure DMSO, it is evident that they are very different. The characteristic peaks of DMSO are absent in the spectrum of the dried layer. This means that it makes sense to compare an FTIR spectra of the GOPS-assisted PEDOT:PSS electrode with that assisted by the DMSO additive. These spectra are presented in Fig. 7(b), where it is shown that the two spectra are practically identical. This suggests that there is no residual trace of the DMSO in the dry layer and it probably only acted during the drying in modifying the morphology of the PEDOT:PSS sample. After these analyses, it was concluded that the electrode with the addition of DMSO is biocompatible, mechanically stable and promising as an electrode for biosensing. However, due to the addition of GOPS the absolute electrical conductivity remains rather low and another way may be investigated if high electric conductivity is needed.

\subsection{Ionic-liquid-based PEDOT:PSS electrode}

Fig. 8(a) shows a visual comparison between a thin and thick IL-based PEDOT:PSS electrode, still on the substrate and in its mold, respectively.

(a)

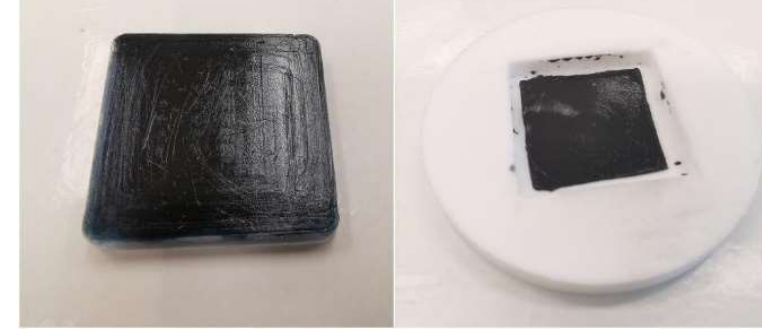

(b)

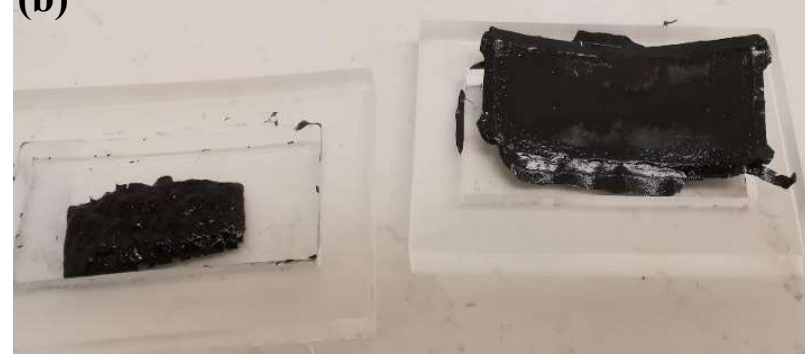




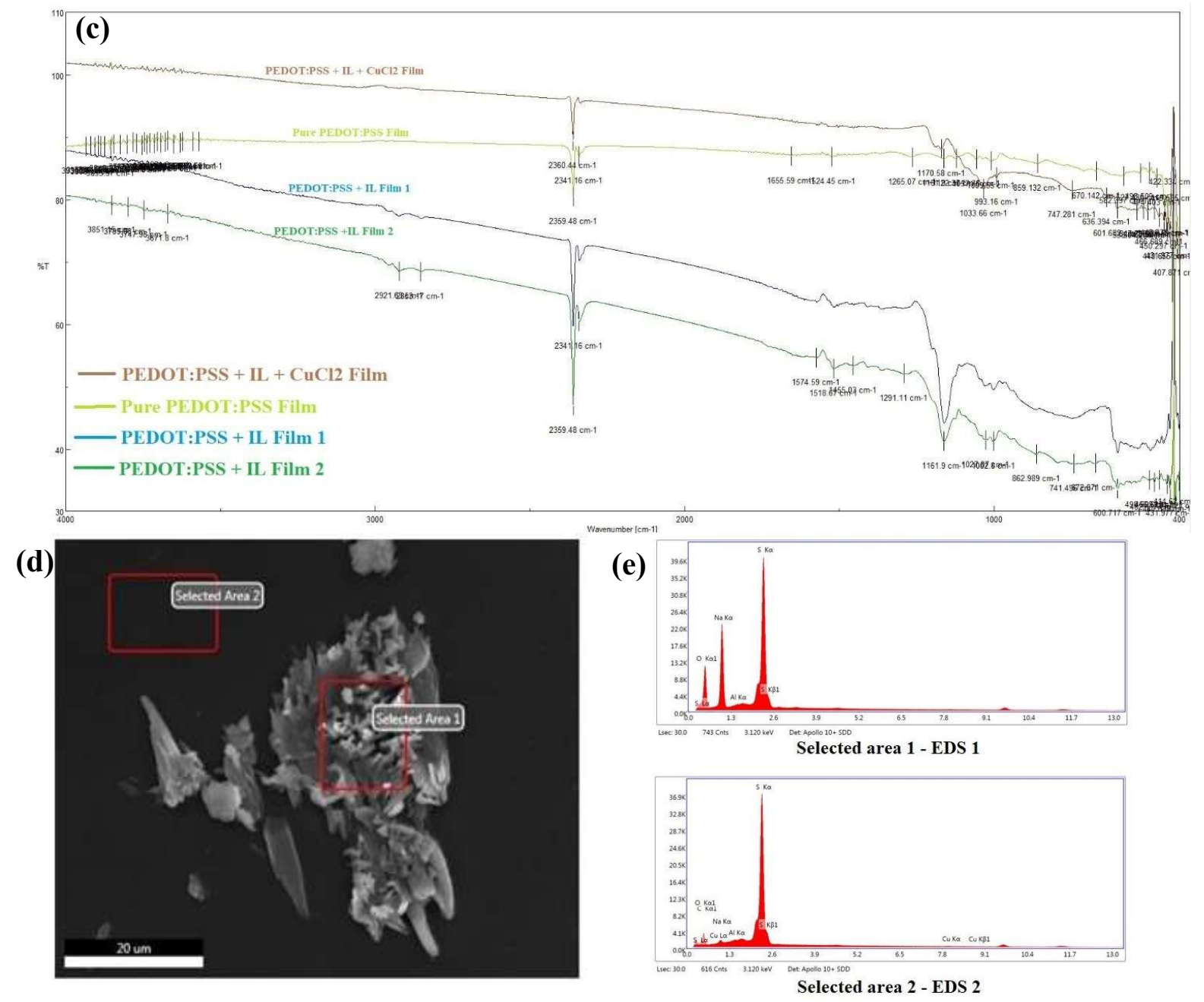

Figure 8. (a) Thin film electrode on a substrate (left) and thick film electrode in a mold (right), (b) difference between thick film with $\mathrm{CuCl}_{2}$ (left) and without (right), (c) FTIR spectra of different PEDOT:PSS films, (d) SEM image with $1500 \mathrm{x}$ magnification, with 2 selected areas for EDS analysis, (e) EDS analysis of the formed crystals

Fig. 8(b) shows for the thick film, once taken out of the mold, a visual comparison between the IL-based PEDOT:PSS electrodes with added $\mathrm{CuCl}_{2}$ (left) and without (right). First of all, Fig. 8(b) shows that once the electrodes are taken out of their preparation device, they shrink, which was not the case for the GOPS-based electrodes. The one with the added $\mathrm{CuCl}_{2}$ salt appears even to shrink more and when thermally treated it showed uneven surface properties. Interestingly, though not the subject of this paper, the latter sample seemed also not to be completely dried, suggesting that water from the preparation solution is entrapped. This is due to the gelation characteristics of $\mathrm{CuCl}_{2}$ and could be of interest for aerogels as is suggested in [37]. FTIR spectra of pure PEDOT:PSS films, those with added ILs and those with adding $\mathrm{CuCl}_{2}$ and ILs are presented in Fig. 8(c). We can see that the spectra are very similar, and the only difference is in the intensity of the peaks. This suggests that there is no significant amount of chemical reactions between the ionic liquid and the PEDOT:PSS (the $\mathrm{CuCl}_{2}$ salt having no significant influence on the chemical morphology), just as it was suggested in [11].

A 1500 magnification shows some formation of crystals in Fig. 8(d). An EDS analysis was done on several different samples, leading to the same conclusion, presented in Fig. 8(e). Here, two EDS spectra are shown, corresponding to two selected areas from Fig. 8(d), i.e. one where the crystals are visible (selected area 1) and one where they are absent (selected area 2). The EDS results in Fig. 8(e) for selected area 2 show that sulfur is present and small amounts of oxygen 
and carbon. Fig. 1 suggests that this corresponds to the PEDOT:PSS structure. Some traces of copper are present as well, which may come from impurities of used samples containing the $\mathrm{CuCl}_{2}$ salt. The EDS results for selected area 1 show peaks for primarily sulfur, oxygen and sodium. The interpretation of this result is suggested to be found in the production process of PEDOT. It appears that typical ways to produce a fully stable PEDOT:PSS is by oxidative chemical polymerization of EDOT in the presence of PSS, where typical oxidizing agents are reported to be $\mathrm{FeCl}_{3}, \mathrm{Fe}(\mathrm{OTs})_{3}$ and $\mathrm{Na}_{2} \mathrm{~S}_{2} \mathrm{O}_{8}[13,14,38]$. Although the sulfur and oxygen peaks would mainly come from the PEDOT:PSS, a combination of sulfur, oxygen and sodium peaks would suggest a crystallization of remaining $\mathrm{Na}_{2} \mathrm{~S}_{2} \mathrm{O}_{8}$, possibly used as an oxidative agent for the production of the purchased PEDOT:PSS dispersion. These results altogether suggest that the used dopants do not interfere with the chemical structure of the electrodes. Table 2 shows what the effect is on the electrical conductivity. The thicknesses of the thick and thin films were rather uniform and found to be around 140 and $20 \mu \mathrm{m}$, respectively.

Table 2. Obtained values for electric properties of the PEDOT:PSS electrodes

\begin{tabular}{lllll}
\hline $\begin{array}{l}\text { Film } \\
\text { thickness }\end{array}$ & IL $[\mathrm{wt} \%]$ & $R_{S}[\Omega]$ & $\varrho[\mu \Omega \mathrm{m}]$ & $\sigma[\mathrm{kS} / \mathrm{m}]$ \\
\hline \hline $140 \mu \mathrm{m}$ & 0 & 21.9 & $3.07 * 10^{3}$ & $0.33 \pm 0.01$ \\
\cline { 2 - 5 } & 15 & 1.05 & 147 & $6.8 \pm 0.1$ \\
& 30 & 0.72 & 101 & $9.9 \pm 0.1$ \\
& 45 & 0.99 & 139 & $7.2 \pm 0.1$ \\
& 60 & 0.54 & 75.6 & $13 \pm 0.1$ \\
\hline \hline $20 \mu \mathrm{m}$ & 0 & - & - & - \\
\hline \hline & 15 & 4.00 & 80 & $13 \pm 3$ \\
& 30 & 1.52 & 30.4 & $33 \pm 6$ \\
& 45 & 1.85 & 37 & $27 \pm 4$ \\
& 60 & 1.63 & 32.6 & $31 \pm 5$ \\
\hline
\end{tabular}

The electric conductivity of the pure PEDOT:PSS film agrees well with that measured in the literature [12]. Even though it could be considered satisfactory [34], this film was so brittle that it could not be used as a cathode (see Fig. 2(b) without GOPS). We measured the electric properties of PEDOT:PSS films with the addition of $\mathrm{CuCl}_{2}$, enhancing in some cases the electric conductivity (see Appendix C). However, the macroscopic morphology was of such an unstable kind that its use as an electrode was physically challenging (see also Fig. 8(b) (left)).

\subsection{PEDOT:PSS as cathode for a prototype cellulose-assisted biosensing device}

In order to demonstrate the applicability of the PEDOT:PSS electrode as a cathode in a biosensing device, we focus more on the DMSO-assisted electrode due to its mechanical stability facilitating good electric connections. As a side note, the IL-doped electrode also appeared to be utilizable as cathode in the biosensing device. It has been proven that during the different healing phases of a wound the skin secretes different fluids, called exudates which have different compositions and are emitted in different quantities according to the woundhealing phase. The configuration used could be calibrated, correlating the current generated by the sensor to the various types of exudate, to become a sensor that continuously monitors the healing of a wound. Using a body fluid simulant as an electrolyte and being completely biocompatible, this configuration could be used directly as a sensor in contact with wounds.

We introduced, for this demonstration, the PEDOT:PSS electrode in the cofiguration presented in Fig. 3(c). We recall that the electrolyte used has a composition of salts and $\mathrm{pH}$ very close to that of the exudates. Fig. 9(a) shows the current response as a function of the absorption of a 
variable quantity of deposited electrolyte of up to $20 \mu \mathrm{l}$. It shows an increase of the current response as the quantity of electrolyte is added. Varying only $5 \mu \mathrm{L}$, there is an evident variation of the current of almost $0.3 \mathrm{~mA}$, which can easily be evaluated with common instruments. Although it is not the purpose of this work, these results show it would be possible to predict the current values for each quantity of liquid introduced and associating it with a very simple system that calculates this current would generate a true sensor capable of constantly monitoring the wounds. A calibration procedure added, this would be a promising biosensor prototype.

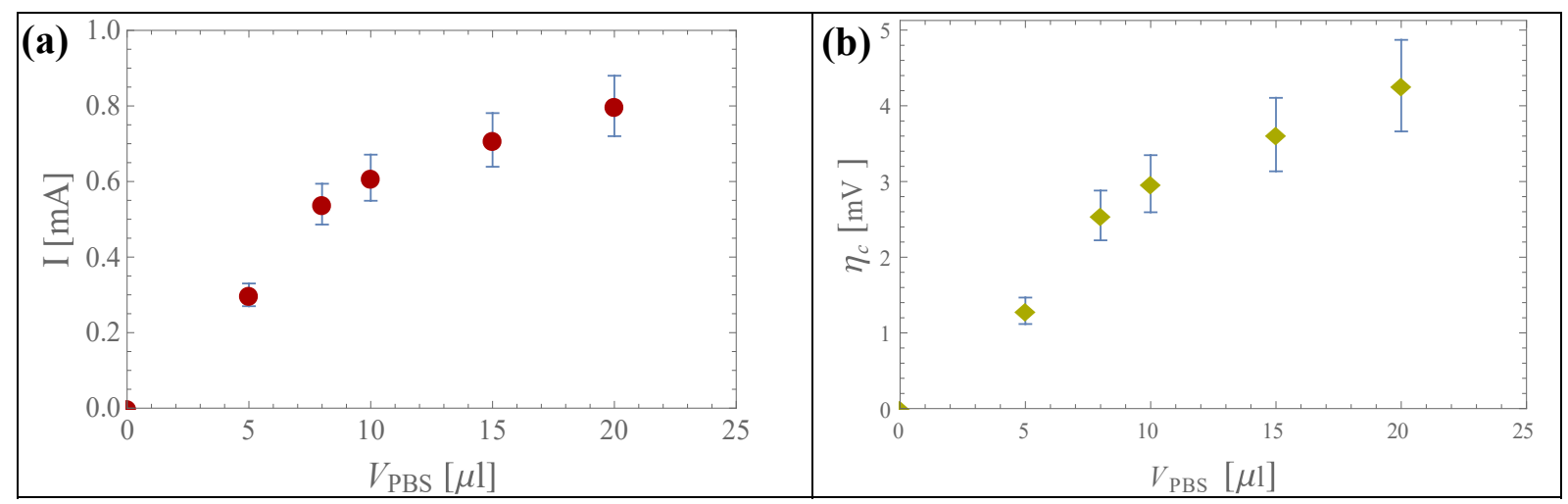

Figure 9. (a) Current response generated by the deposition of different quantities of PBS, (b) Overpotential due to oxygen diffusion through the PEDOT:PSS cathode corresponding to the current responses generated by the deposited PBS electrolyte.

The calibration procedure and electrode efficiency would depend on energy losses of the cathode and for this purpose we estimated the overpotential caused by oxygen diffusion through the cathode pores. There are energy losses, such as electrolyte overpotentials, but the focus here is on the cathode reusability. The overpotential from Eq. (4) as a function of the amount of deposited electrolyte (related to the measured current in Fig. 9(a)) is shown in Fig. 9(b). It can be shown that, although an increasing amount of deposited electrolyte enhances the overpotential, it remains of the order of $\mathrm{mV}$, which is significantly less than the working potential. It is expected that the reusability prospectives are rather promising. More in-depth analysis of the electrode would check this perspective. First, the correct operation of the biosensor can be evaluated by looking to the whole assembly. After every discharge test was done, the sensors were disassembled, visually inspected and then further analyzed as follows. A SEM image obtained of the zinc electrode is shown in Fig. 10(a) and (c).

(a)

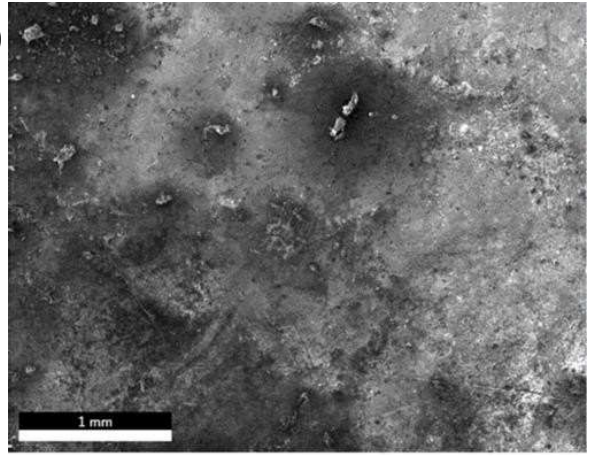

(b)

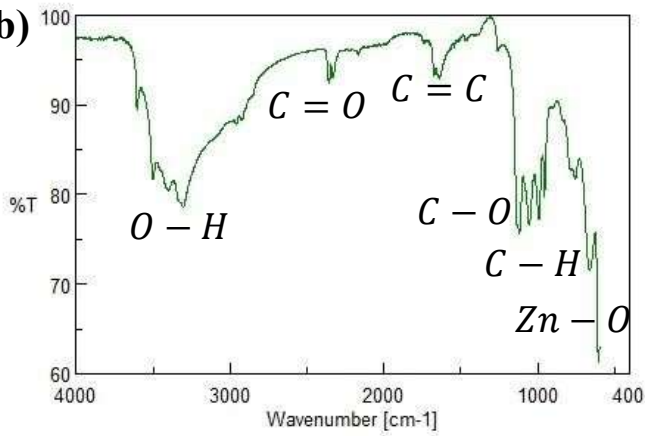


(c)

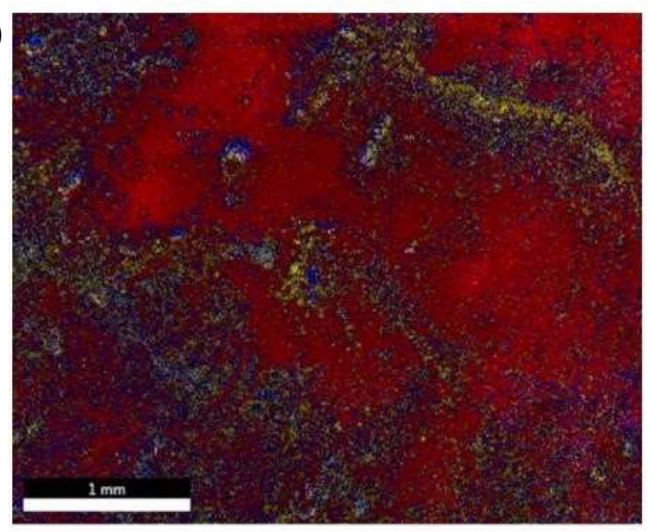

$10 \%$ ZnK/CIK/O K/K KJSiK R20413 Pixels

$59 \%$ ZnK/O K/CIK/K K (121614 Pixels)

$4 \%$ Unallocated (7848 Pixels)

$1 \%$ aINO K/ZnK/K N/SiK (1226 Pixels)

$5 \%$ ZnKJO K/SiK/K K/CIK (9317 Pixels)

$22 \%$ ZnK/O K/CIK/K KJSIK (44288 Pixels)

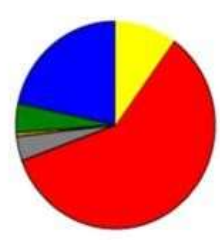

Figure 10. (a) SEM image of the zinc surface after the discharge, (b) FTIR spectra of the served Zn-electrode residue, (c) color map of the surface of the zinc electrode after the discharge obtained with SEM-EDX

Fig. 10(a) shows a non-smooth image indicating that the zinc foil has undergone changes. In order to determine the composition of the material formed on the Zn-electrode, a FTIR analysis of the served anode electrode was done. The resulting spectrum is shown in Fig. 10(b). The peak around $650 \mathrm{~cm}^{-1}$ is reported to be attributed to $\mathrm{Zn}-\mathrm{O}$ stretching [39]. This is a strong indication that indeed $\mathrm{ZnO}$ is formed during the use of the sensor. This is confirmed by a color map from SEM-EDX measurements in Fig. 10 (c), where, besides $\mathrm{Cl}$ and $\mathrm{K}$ elements coming most probably from the PBS electrolyte adsorption, mainly $\mathrm{Zn}$ and $\mathrm{O}$ elements are present (red and blue regions). The wide peak around $3300 \mathrm{~cm}^{-1}$ in Fig. $10(\mathrm{~b})$ can be attributed to O-H stretching, probably originating from adsorbed water [39]. All the other peaks are suggesting an organic molecule $\left(\mathrm{C}=\mathrm{O}\right.$ stretching around $2200 \mathrm{~cm}^{-1}, \mathrm{C}=\mathrm{C}$ stretching between 1600 and 1700 $\mathrm{cm}^{-1}, \mathrm{C}-\mathrm{O}$ stretching around $1200 \mathrm{~cm}^{-1}$ and $\mathrm{C}-\mathrm{H}$ bending around $1000 \mathrm{~cm}^{-1}$ ) [40]. Given that the analyzed material was in contact with filter paper, this organic molecule shown in the spectrum could well be cellulose from the separator. Fig. 11(a) shows a SEM image of the separator.

(a)

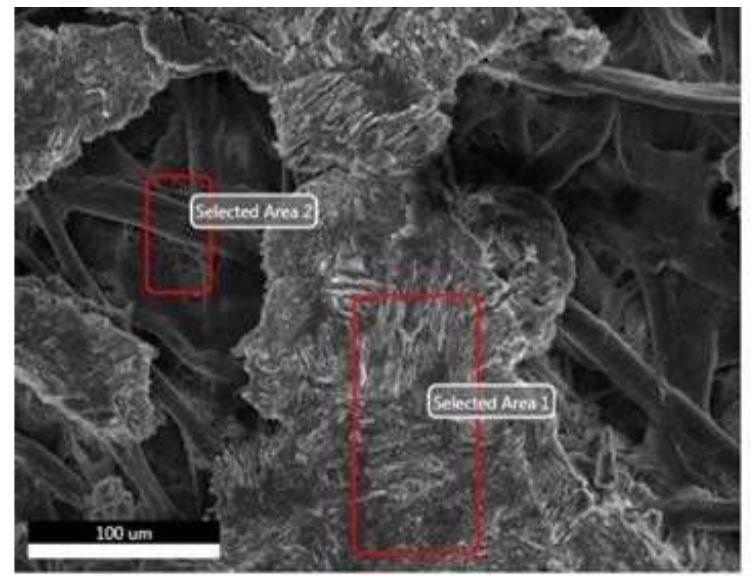

(b)
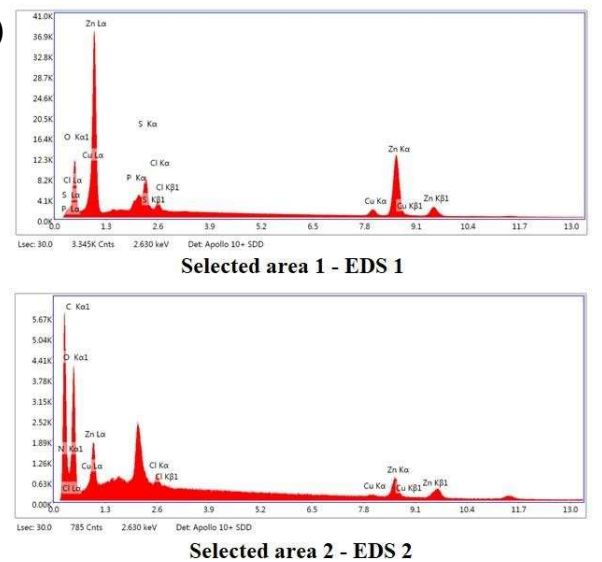

Figure 11. (a) SEM image of the separator, (b) EDS analysis of the filter paper

Fig. 11(a) shows two selected areas, i.e. selected area 1, where crystals are visible and selected area 2, where fibres are visible. Further EDS analysis of these selected areas is presented in Fig. 11(b). The fiber-like structure of the Selected Area 2 suggests that this is the filter paper, indicated by a predominant presence of carbon and oxygen, the main components of cellulose. On the other hand, the crystals formed in Selected Area 1 are most probably $\mathrm{ZnO}$, as the EDS spectrum shows predominantly $\mathrm{Zn}$ and oxygen. This is further proof that the reaction is occurring and that the exchange of ions is present. Finally, Fig. 12 shows EDS images of the PEDOT:PSS 
after discharge, comparing it to the one before.
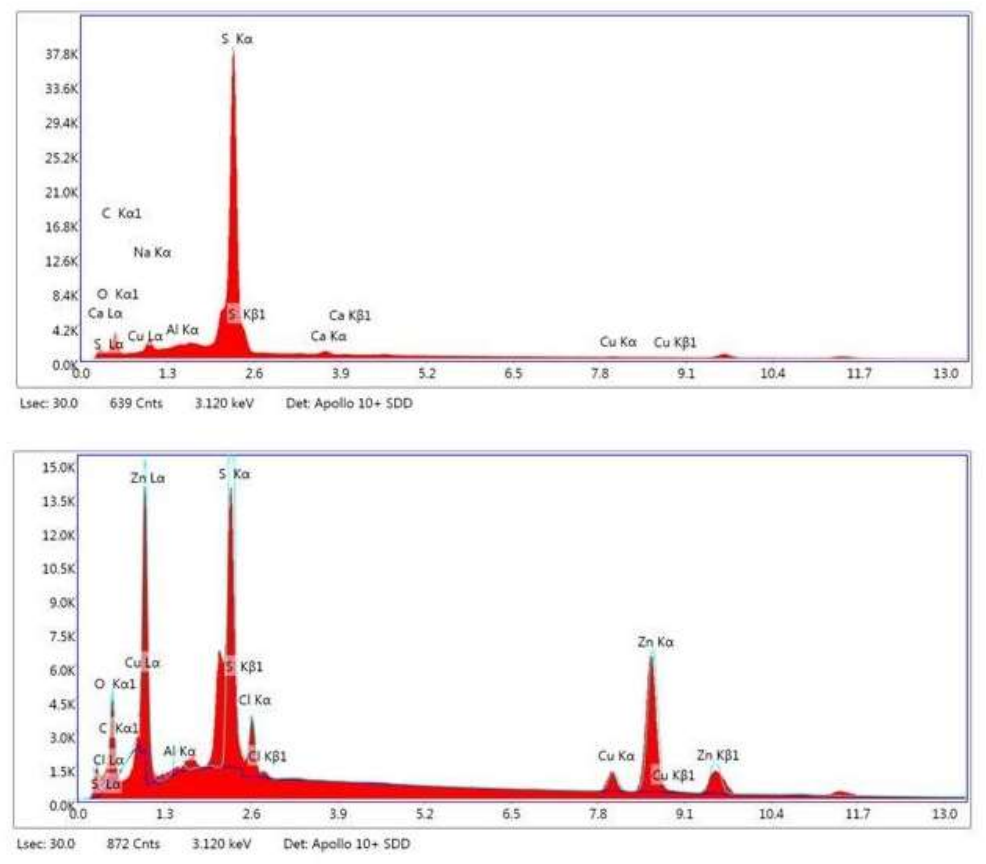

Figure 12. EDS spectra of the PEDOT:PSS spectra (a) before using the sensing device and (b) after complete discharge

Some traces of $\mathrm{K}, \mathrm{Cu}$ and $\mathrm{Cl}$ are observed in Fig. 12. The $\mathrm{K}$ and $\mathrm{Cl}$ traces may come from the PBS, indicating some adsorption of $\mathrm{KCl}$ salt on the PEDOT:PSS, and the copper may come from left impurities from the tests with $\mathrm{CuCl}_{2}$ addition. Fig. 12 clearly shows that, comparing the EDS spectra before the use of the PEDOT:PSS electrode and after its use, the oxygen and zinc content in the latter are substantially increased. This again shows that $\mathrm{ZnO}$ has been deposited on the PEDOT:PSS electrode, suggesting that the predominant reactions are indeed those between atmospheric oxygen and zinc as illustrated in Fig. 3(c), where PEDOT:PSS mainly serves as an electrode and, in aqueous medium, as a catalyst for the oxygen reduction reaction. This observation appeared to be the case for both the DMSO-assisted and the IL-doped PEDOT:PSS electrodes. Although not observed within the configuration of this work and apparently not significant for the correct operation of the sensing device, it should be kept in mind that there are indications that in playing the role of a catalyst, the PEDOT:PSS electrode can undergo an oxidation [32,33]. Change in the chemical structure of the PEDOT:PSS has not been significantly observed in this work and is also confirmed by the cyclic voltammetry tests. This could possibly indicate several reuses of the PEDOT:PSS electrode for the sensing device. The analysis shows that most probably this device is indeed functioning correctly and demonstrates, although further development is of course necessary, the possible application as a long-term monitoring sensing device. Preliminary tests have shown that this device (with currents of the order of $0.2 \mathrm{~mA}$ ) could function around $20 \mathrm{~h}$. However, considering that this lifetime is due to the consumption of the $\mathrm{Zn}$ anode, the PEDOT:PSS cathode appearing to be reutilisable, one might consider low-cost easily replacable anodes, increasing thereby considerably the biosensor's lifetime. All in all, the present work already shows good results for operable PEDOT:PSS hydrogels as cathode for short-term biosensor monitoring, and in some medical applications it might just be enough.

\section{Conclusions}

The primary aim of this research project was to characterize the influence of electrode 
preparation and formulation on the electrical conductivity and chemical composition of a PEDOT:PSS electrode in view of their application for sensing devices and energy storage systems. Moreover, it was to investigate whether this electrode undergoes any changes during its use as a cathode in a sensing device. The 1-butyl-3-methylimidazolium octyl sulfate ionic liquid proved to be crucial for film formation as a stabilizer, although the electrodes did not maintain their initial size over time, which can make tailored production difficult. The ionic liquid also served well as a significant electrical conductivity enhancer, achieving films with conductivities as high as $30000 \mathrm{~S} / \mathrm{m}$, two orders of magnitude larger than pure PEDOT:PSS. Although some electric conductivity enhancement was registered for films with added copper(II)-chloride, their applicability was considered challenging due to their unstable mechanical morphologies. FTIR analysis suggested that during the formation process of the films no significant changes in the chemical structure of the PEDOT:PSS hydrogels was observed. The electrodes that were based on GOPS addition were more robust and their dimensions where easier to control. However, even though organic solvant additives increased substantially their electric conductivity, it was in absolute still much lower than for the electrode based on ionic liquid doping. This work has shown that the use of ionic liquids as a secondary doping gives much better results in terms of electric conductivity, but in return the electrodes were less robust. Finally, it was demonstrated that with minimal adaptation, the PEDOT:PSS electrode could be successfully integrated in a (bio)sensing device for electrolytes. It was also shown that as a cathode, the PEDOT:PSS electrode retained its chemical composition. Indeed, EDS and FTIR analyses suggested that after its use in the sensing device no significant changes in the chemical structure was observed and that the primary reactions occured between $\mathrm{Zn}$ and oxygen, which suggests the possible reutilization of such an electrode. Furthermore cyclic voltammetry showed a nearly fully reversibility for the PEDOT:PSS electrodes in se, indicating small degradation and a longer lifetime. These results suggest promising applications for PEDOT:PSS electrodes, especially in (bio)sensing devices. Applications in power supply, such as batteries and supercapacitors, may also be interestingly feasible fields to investigate.

\section{Acknowledgements}

This research has received funding from the European Union H2020 Programme under Grant agreement number 785219: Graphene Flagship. G. Wallaert from the "Materials engineering, characterization, synthesis and recycling" group at ULB is thanked for his technical assistance for the SEM(EDS) images and profilometry tests.

\section{Declarations}

Funding. European Union H2020 Programme under grant number 785219: Graphene Flagship Conflict of interest. No conflict of interest.

Availability of data and transparency. All data are in the article.

Code availability. Not applicable.

\section{Appendix}

\section{A. Reduction-oxydation of PEDOT}

In a metal-polymer battery, in addition to acting as a catalyst for metal-air reactions, the polymer cathode can undergo changes during the discharge process [33]. In particular, it has been proven that oxidation-reduction reactions take place on the surface of the polymer. Fig. A1 shows an image from [33] which illustrates the PEDOT in oxidized and reduced forms. Cyclic voltammetry tests were performed to analyze the reversibility of such reactions. 

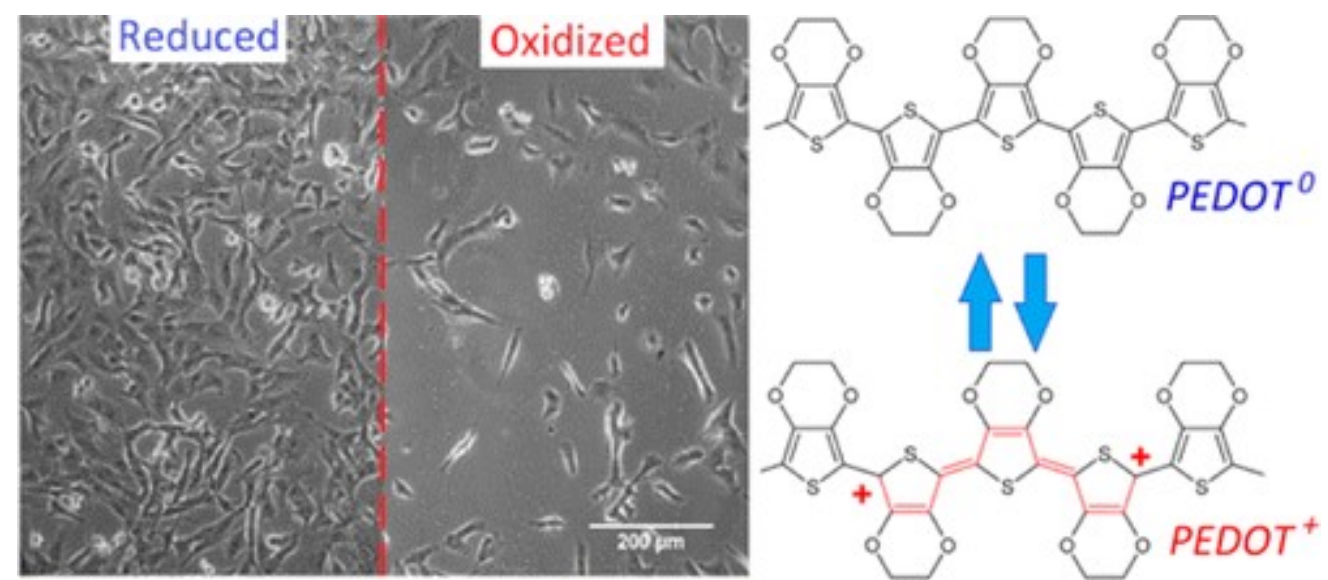

Fig. A1. Reversible reduction and oxidation of PEDOT from [28]

\section{B. Cyclic voltammogram of graphite}

Fig, B1 clearly shows the highly irreversible character of the graphite electrode in contrast to that of the PEDOT:PSS hydrogel. We can estimate $\frac{\left|i_{a}\right|}{\left|i_{c}\right|} \approx 5$.

\section{Standard Graphite electrode}

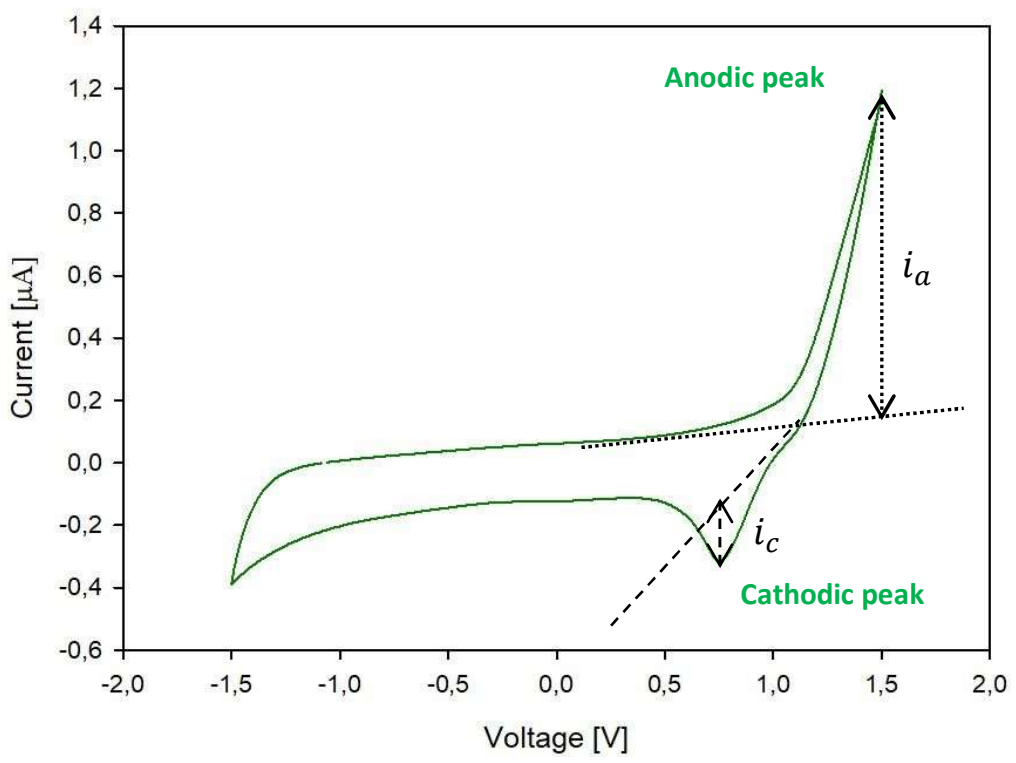

Fig B1: Cyclic voltammogram of a graphite electrode

\section{Electric properties for $\mathrm{CuCl}_{2}$-doped PEDOT:PSS hydrogel films}

The electric conductivities of the PEDOT:PSS films with $\mathrm{CuCl}_{2}$ salt additions are shown in Table C1.

Table $\mathrm{C} 1$ : Electric properties of $\mathrm{CuCl}_{2}$ doped PEDOT:PSS films containing $45 \mathrm{wt} \% \mathrm{IL}$.

\begin{tabular}{lllll}
\hline Film type & $\mathrm{CuCl}_{2}[\mathrm{~mol} \%]$ & $R_{S}[\Omega]$ & $\varrho[\mu \Omega \mathrm{m}]$ & $\sigma[\mathrm{kS} / \mathrm{m}]$ \\
\hline \hline $140 \mu \mathrm{m}$ & 0 & 0.99 & 139 & 7.2 \\
& 1 & 0.52 & 72.8 & 14 \\
& 3 & 0.73 & 102 & 9.8
\end{tabular}




\begin{tabular}{lllll} 
& 5 & 0.63 & 88.2 & 11 \\
\hline \multirow{4}{*}{$20 \mu \mathrm{m}$} & 0 & 1.85 & 37 & 27 \\
& 1 & 2.04 & 40.8 & 25 \\
& 3 & 2.56 & 51.2 & 20 \\
& 5 & 2.60 & 52 & 19 \\
\hline
\end{tabular}

\section{References}

[1] C. André da Costa, C.F. Pasluosta, B. Eskofier, D. Bandeira da Silva, R. da Rosa Righi, Internet of Health Things: Toward Intelligent Vital Signs Monitoring in Hospital Wards, Artificial Intelligence in Medicine 89 (2018) 61-69.

[2] X. Jia, Y. Yang, C. Wang, C. Zhao, R. Vijayaraghavan, D.R. MacFarlane, M. Forsyth, G.G. Wallace, Biocompatible ionic liquid-biopolymer electrolyte-enabled thin and compact Magnesium-air batteries, ACS Appl. Mater. Interfaces 6(23) (2014) 21110-21117.

[3] H. Shirakawa, E.J. Louis, A.G. MacDiarmid, C.K. Chiang, A.J. Heeger, Synthesis of electrically conducting organic polymers: Halogen derivatives of polyacetylene, $(\mathrm{CH}) \mathrm{x}, \mathrm{J}$. Chem. Soc. Chem. Commun. 16 (1977) 578-580.

[4] D. Fichou, G. Horowitz, Molecular and polymer semiconductors, conductors, and superconductors: overview, Encycl. Mater. Sci. Technol. (2001) pp. 5748-5757.

[5] C.K. Chiang, C.R. Fincher Jr., Y.W. Park, A.J. Heeger, H. Shirakawa, E.J. Louis, S.C. Gau, A.G. MacDiarmid, Electrical conductivity in doped polyacetylene, Phys. Rev. Lett. 39(17) (1977) 1098-1101.

[6] P. Chandrasekhar, Conducting polymers, fundamentals and applications, 1st edition. Springer, 1999.

[7] J. Tarver, M. Sezen-Edmonds, J. E. Yoo, Y.-L. Loo, Organic electronic devices with waterdispersible conducting polymers, in Comprehensive Nanoscience and Technology, 2011, pp. 413-446.

[8] F. Wolfart, B.M. Hryniewicz, M.S. Góes, C.M. Corrêa, R. Torresi, M.A.O.S. Minadeo, S.I. Córdoba de Torresi, R.D. Oliveira, L.F. Marchesi, M. Vidotti, Conducting polymers revisited: applications in energy, electrochromism and molecular recognition, J. Solid State Electrochem. 21(9) (2017) 2489-2515.

[9] Y. Wen, J. Xu, Scientific importance of water-processable PEDOT-PSS and preparation, challenge and new application in sensors of its film electrode: a review," J. Polym. Sci. Part A Polym. Chem. 55(7) (2017) 1121-1150.

[10] M. Döbbelin, R. Marcilla, M. Salsamendi, C. Pozo-Gonzalo, P.M. Carrasco, J.A. Pomposo, D. Mecerreyes, Influence of ionic liquids on the electrical conductivity and morphology of PEDOT:PSS films, Chem. Mater. 19(9) (2007) 2147-2149.

[11] M.U. Hassan, Y.C. Liu, K. ul Hasan, H. Butt, J.F. Chang, R.H. Friend, Highly efficient PLEDs based on poly(9,9-dioctylfluorene) and Super Yellow blend with $\mathrm{Cs}_{2} \mathrm{CO}_{3}$ modified cathode, Appl. Mat. Today 1(1) (2015) 45-51.

[12] A. Hakansson, S. Han, S. Wang, J. Lu, S. Braun, M. Fahlman, M. Berggren, X. Crispin, S. Fabiano, Effect of (3-Glycidyloxypropyl) trimethoxysilane (GOPS) on the electrical properties of PEDOT:PSS films, J. Polymer Sci. B: Polymer Physics 55 (2017) 814-820.

[13] L. Groenendaal, F. Jonas, D. Freitag, H. Pielartzik, J.R. Reynolds, Poly(3,4ethylenedioxythiophene) and its derivatives: past, present, and future, Adv. Mater. 12(7) (2000) 481-494.

[14] W. Lövenich, PEDOT-properties and applications, Polym. Sci. - Ser. C 56(1) (2014) 135143.

[15] H. Shi, C. Liu, Q. Jiang, J. Xu, Effective approaches to improve the electrical conductivity of PEDOT:PSS: a review, Adv. Electron. Mater. 1(4) (2015) 1-16.

[16] J.M. Gomes, S.S. Silva, R.L. Reis, Biocompatible ionic liquids: fundamental behaviours 
and applications, Chem. Soc. Rev. 48(15) (2019) 4317-4335.

[17] O.P. Dimitriev, D.A. Grinko, Yu.V. Noskov, N.A. Ogurtsov, A.A. Pud, PEDOT:PSS films - Effect of organic solvent additives and annealing on the film conductivity, Synthetic Metals 159 (2009) 2237-2239.

[18] C.S. Pathak, J.P.Singh, R.Singh, Effect of dimethyl sulfoxide on the electrical properties of PEDOT:PSS/n-Si heterojunction diodes, Current Appl. Phys. 15 (2015) 528-534.

[19] Y. Wang, C. Zhu, R. Pfattner, H. Yan, L. Jin, S. Chen, F. Molina-Lopez, F. Lissel, J. Liu, N.I. Rabiah, Z. Chen, J.W. Chung, C. Linder, M.F. Toney, B. Murmann, Z. Bao, A highly stretchable, transparent, and conductive polymer, Sci. Adv. 3(3) (2017) 1-11.

[20] B. Kim, J.U. Hwang, E. Kim, Chloride transport in conductive polymer films for an n-type thermoelectric platform, Energy Environ. Sci. 13 (2020) 859-867.

[21] P. Gu, M. Zheng, Q. Zhao, X. Xiao, H. Xue, H. Pang, Rechargeable zinc-air batteries: A promising way to green energy, J. Mater. Chem. A 5(17) (2017) 7651-7666.

[22] P. Sapkota, H. Kim, Zinc-air fuel cell, a potential candidate for alternative energy, J. Ind. Eng. Chem. 15(4) (2009) 445-450.

[23] D.K. Schroder, Semiconductor material and device characterization, 3rd edition. John Wiley \& Sons, 2006.

[24] R. Leach, Surface topography measurement instrumentation, 2nd edition. Elsevier, 2014.

[25] D.J. Stokes, Principles and practice of variable pressure/environmental scanning electron microscopy (VP-ESEM). 1st edition. John Wiley \& Sons, 2008.

[26] R.H. Geiss, EDS: Energy-Dispersive X-Ray Spectroscopy, in Encyclopedia of Materials Characterization, 1992, pp. 120-134.

[27] F. Zhao, T.J. Armstrong, A.V. Virkar, Measurement of $\mathrm{O}_{2}-\mathrm{N}_{2}$ effective diffusivity in porous media at high temperatures using an electrochemical cell, Journal of the Electrochemical Society 150 (2003) A249-A256.

[28] H. Machrafi, Universal relation between the density and the viscosity of dispersions of nanoparticles and stabilized emulsions, Nanoscale 12 (2020) 15081-15101.

[29] H. Machrafi, Extended Non-Equilibrium Thermodynamics: From Principles to Applications in Nanosystems, Taylor \& Francis Group, London, 1st edn (2019).

[30] H. Machrafi, G. Lebon, Fluid flow through porous and nanoporous media within the prisme of extended thermodynamics: emphasis on the notion of permeability, Microfluidics and Nanofluidics 22 (2018) 65.

[31] Private communication with Pr X. Crispin from the "Laboratory of Organic Electronics, Department of Science and Technology, Linköping University", stating that micro- to nanoporous scales are expected, so that we estimate the pore size by $1 \mu \mathrm{m}$.

[32] C. Zhu, J. Zhai, D. Wen, S. Dong, Graphene oxide/polypyrrole nanocomposites: one-step Electrochemical doping, coating and synergistic effect for energy storage, J. Mater. Chem. 22(13) (2012) 6300-6306.

[33] M. Marzocchi, I. Gualandi, M. Calienni, I. Zironi, E. Scavetta, G. Castellani, B. Fraboni, Physical and electrochemical properties of PEDOT:PSS as a tool for controlling cell growth 7(32) (2015) 17993-18003.

[34] B. Lu, H. Yuk, S. Lin, N. Jian, K. Qu, J. Xu, X. Zhao, Pure PEDOT:PSS hydrogels, Nature Comm. 10 (2019) 1043.

[35] M. Yang, Y. Zhang, H. Zhang, Z. Li, Characterization of PEDOT:PSS as a biocompatible conductive material, 10th IEEE International Conference on Nano/Micro Engineered and Molecular Systems, Xi'an, China, 2015, pp. 149-151.

[36] V. Karagkiozaki, P.G. Karagiannidis, M. Gioti, P. Kavatzikidou, D. Georgiou, E. Georgaraki, S. Logothetidis, Bioelectronics meets nanomedicine for cardiovascular implants: PEDOT-based nanocoatings for tissue regeneration, Biochimica et Biophysica Acta 1830(9) (2013) 4294-4304.

[37] G. Chen, R. Rastak, Y. Wang, H. Yan, V. Feig, Y. Liu, Y. Jiang, S. Chen, F. Lian, F. 
Molina-Lopez, L. Jin, K. Cui, J.W. Chung, E. Pop, C. Linder, Z. Bao, Strain- and strain-rateinvariant conductance in a stretchable and compressible 3D conducting polymer foam, Matter 1(1) (2019) 205-218.

[38] S. Kirchmeyer, K. Reuter, Scientific importance, properties and growing applications of poly(3,4-ethylenedioxythiophene)," J. Mater. Chem. 15(21) (2005) 2077-2088.

[39] K.S. Babu, A.R. Reddy, C. Sujatha, K.V. Reddy, A.N. Mallika, Synthesis and optical characterization of porous ZnO, J. Adv. Ceram. 2(3) (2013) 260- 265.

[40] Sigma Aldrich, IR Spectrum Table \& Chart: https://www.sigmaaldrich.com/technicaldocuments/articles/biology/ir-spectrum- table.html. Accessed 26 February 2021. 